\title{
Higher Dimensional Dark Energy and Phantom Energy Dilation Brans-Dicke Multiverse with Positive Cosmological Constant
}

\author{
*H. R. Ghate ${ }^{1}$ and Arvind S. Patil ${ }^{2}$ \\ ${ }^{I}$ Department of Mathematics, Jijamata Mahavidyalaya, Buldana (India) - 443001 \\ ${ }^{2}$ Department of Mathematics, Jijamata Mahavidyalaya, Buldana (India) - 443001
}

\begin{abstract}
The present study deals with higher dimensional dilaton-Brans-Dicke cosmology where the multiverse is filled with dark energy or phantom energy having a positive cosmological constant and which contains infinite singularities with eternally Big Rip. It is the extension of 4-dimensional cosmological model discussed by El-Nabulsi Ahmad Rami (2010).
\end{abstract}

Keywords: Anisotropic Generalised Chaplygin gas, Dilation Brans -Dicke scalar field, Dynamical cosmological constant. Dark energy. Phantom energy. Big Rip. Multiverse. Higher dimensions. Dark Energy, Perfect Fluid, Bianchi type-IX Universe.

\section{Introduction:}

The Observations [1-6] claims that the universe at large scale is homogeneous and isotropic. Many cosmological observations like Ia Supernova (SNe Ia) and results from Wilkinson Microwave Anisotropic Probe (WMAP) [7-8] and Chandra X-ray observatory [9] etc. have conclude that universe is in accelerated expansion phase. It is believed that this expansion of universe is due to the presence of dark energy. The universe density consists of $4 \%$ visible matter, $76 \%$ dark energy and $20 \%$ dark matter .

In 1998, from the experiments of two different teams of researchers, as the universe is accelerating indicating that the cosmological constant wasn't just zero as expected but had to have a very slight positive value. With this concept of positive cosmological constant, the theory of dark energy has introduced. There are other causes of accelerating universe such as quintessence but the cosmological constant is in most respects the simplest solution. The unusual large value of cosmological constant arising out of the spontaneously broken field theoretic vacuum leads to the search for alternative dynamical dark energy models. To solve the quintessence trouble, lot of works have been done and a new class of cosmological models characterized by exotic type of scalar field have been conjectured for modeling the dark energy in terms of sub-negative pressure. Various models are studied in this respect specially Viscous fluid, K-essence, Chaplygin gas with equation of state $p=-K \rho^{-1}, \rho>0, K \in R$ [10], exotic matters with generalized equation of state $p=A \rho-B \rho^{a}$, $A, B, a \in R$ [11], generalized Chaplygin gas model with equation of state is $p=-K \rho^{-a}, \rho>0$, $0<a \leq 1$ [12], Holographic dark energy and so on [13-17].

The unification of gravity with other fundamental forces in nature is still challenging problem today. Modern developments of superstring theory [18] and Young Hiper Mills super gravity in its field theory limit need higher dimensional space times. Hence in recent years, researchers are interested in theories with higher dimensional space times. The experimental detection of time variation of fundamental constant could provides strong evidence for existence of extra dimensions, was suggested by Alvarez et. al. [19] Randjbar-Daemi et. al. [20], Marciano [21]. The theory in which gravitation and electromagnetism could be unified in single geometrical structure was proposed by Kaluza [22] and Klein[23]. Thiry[24] and Jordan [25] generalized the Kaluza Klein idea to consider the coefficient of fifth coordinate constant. Number of authors [26-34] have studied the physics of the universe in higher dimensional space times.

Brans-Dicke [35] suggested a scalar tensor theory of gravitation which is developed by various modifications in general relativity called as Brans - Dicke theory of gravitation. This theory is the scalar tensor theory in which the gravitational interaction involves as scalar field $\phi$ and the metric tensor $g_{i j}$. The scalar field $\phi$ has the dimension of the inverse of the gravitational constant ' $G$ ', where $\phi$ is accepted to satisfy a scalar wave equation whose source is all the matter in universe. The varying gravitational constant, the non-minimal coupling between the scalar field and geometry, compatibility with equivalence principle, Mach's principle and Dirac's large number hypothesis are some important characteristics of this theory [36]. Naria [37], Belinskii and Khalantnikov [38], Reddy and Rao [39], Banergee and Santos [40], Singh et.al. [41], Shriram [42], Shriram and Singh [43], Beram et. al. [44], Reddy [45], Reddy et.al. [46], Adhav et. al. [47], Rao et. al. [48] are some authors who have investigated several aspects of this theory. 
Recently El-Nabulsi [49-57] introduced the equation of state in a particular Brans-Dicke cosmology controlled by generalized chaplygin gas is given by,

$$
p=3 \eta a^{m} \rho^{n}-\rho, \quad(\eta, m, n) \in R
$$

The cosmological constant and dark energy models provide a possible mechanism to allow for an accelerating universe. Restricting ourselves to constant $\omega$, which treats as a flourishing conjecture. The mathematical forms of the cosmological constant and the dilation scalar field should be fixed as follows :

(1) The cosmological constant $\Lambda$ is considered as a function of matter density $\rho$ and scalar field $\phi$ given as $\Lambda \phi=4 \pi \varepsilon \rho, \varepsilon \in R$. It is noted that the effective gravitational constant in the theory is $|G| \alpha \phi^{-1}$, which implies $\Lambda \alpha G \rho$, which is obtained in many cosmological theories exploring the decaying vacuum energy densities [59]

(2) We conjecture that $\phi=\xi a,{ }^{\alpha}(\xi, \alpha) \in R$, as for dilation scalar field.

The corresponding modified Einstein field equations are

$$
\begin{aligned}
& R_{i j}-\frac{1}{2} g_{i j} R=\Lambda g_{i j}-8 \pi G T_{i j}-\frac{\omega}{\phi}\left(\phi_{, i} \phi_{, j}-\frac{1}{2} g_{i j} \phi_{, p} \phi^{p}\right)-\frac{1}{\phi}\left(\phi_{i ; j}-g_{i j} \mathrm{p} \phi\right) \\
& \mathrm{p} \phi=\phi_{; i}^{; i}=\frac{8 \pi T^{(\text {matter })}}{2 \omega+3} \\
& T_{; i}^{i j}=0,
\end{aligned}
$$

where $R_{i j}$ is a Ricci tensor, $R$ is a Ricci scalar, $T_{i j}$ is an energy momentum tensor.

In this paper, dark energy and phantom energy dilaton Brans-Dicke 4-dimmensional multiverse with positive cosmological constant studied by El-Nabulsi (2010) [58] is extended in higher dimensional spacetimes.

\section{Field Equations:}

The higher dimensional FRW metric for homogeneous and isotropic flat universe is given by,

$$
d s^{2}=-d t^{2}+a^{2}(t)\left(d x^{2}+d y^{2}+d z^{2}+d v^{2}\right),
$$

where $a(t)$ is scale factor and $t$ represents the cosmic time, $v$ is the fifth dimension in the space time. Here 0 , $1,2,3,4$ represent the variables $t, x, y, z, v$ respectively.

The energy momentum tensor in the early universe is given by,

$$
T_{i j}=(\rho+p) u_{i} u_{i}+g_{i j} p,
$$

where $\rho$ is the energy density, $g_{i j}$ is the metric tensor, $u_{i}$ is the four velocity of fluid which satisfy the condition $u^{i} u_{i}=0$ for $i=1,2,3,4$ and $u^{0} u_{o}=-1$.

Using equations (5) and (6), the matter tensor is given by

$$
T_{j}^{i}=\operatorname{diag} .(-\rho, p, p, p, p) .
$$

Using (6), (7), (8) for the component $(0,0)$, equation (2) takes the form

$$
H^{2}+\frac{2}{3} H \frac{\dot{\phi}}{\phi}=\frac{\omega}{12} \frac{\dot{\phi}^{2}}{\phi^{2}}+\frac{8 \pi \rho}{6 \phi}+\frac{\Lambda}{6}
$$

where $H=\frac{\dot{a}}{a}$ is the Hubble parameter and $\rho$ is the matter density.

Adopting the equation of state

$$
p=(\gamma-1) \rho, \text { where } \gamma \text { is constant, }
$$

the modified Klein-Gordon equation is then

$$
\ddot{\phi}+3 H \dot{\phi}+\frac{8 \pi}{2 \omega+3}\left[(4 p-\rho)-\frac{\phi \Lambda}{4 \pi}\right]=0 \text {. }
$$

The conservation equation of stress-energy tensor $T_{; i}^{i j}=0$ becomes

$$
\dot{\rho}+4 H \gamma \rho=0
$$


which on simplification gives

$$
\rho=\left[K-12(1-n) \eta \frac{a^{m}}{m}\right]^{\frac{1}{1-n}}
$$

where $K$ is the constant of integration.

At $t=0, a=a_{0}=1, \rho=\rho_{0}=1$, equation (12) gives $\quad \rho=\left[1+\frac{12(1-n) \eta}{m}-\frac{12(1-n) \eta a^{m}}{m}\right]^{\frac{1}{1-n}}$.

From equation (9), putting $\phi=\xi a,{ }^{\alpha}(\xi, \alpha) \in R$ we get

$$
\left[1+\frac{2}{3} \alpha-\frac{\alpha^{2}}{12} \omega\right] H^{2}=\frac{8 \pi+4 \pi \varepsilon}{6 \xi a^{\alpha}}\left[1+\frac{12(1-n) \eta}{m}-\frac{12(1-n) \eta}{m} a^{m}\right]^{\frac{1}{1-n}} .
$$

On simplification of (11) we get,

$$
\omega=\frac{-4[4 p-\rho(1+\xi)]}{\xi \alpha\left[(\alpha+2) \dot{a}^{2} a^{\alpha-2}+a^{\alpha-1} \ddot{a}\right]^{-\frac{3}{2}} .}
$$

To get reasonable solution put $n=0$ and $m=\alpha$ in the equation (14)

$$
\begin{aligned}
& H^{2}=\frac{(8 \pi+4 \pi \varepsilon)}{6 \xi} \frac{\left(1+\frac{12 \eta}{\alpha}\right)}{\left(1+\frac{2 \alpha}{3}-\frac{\alpha^{2}}{12} \omega\right)} a^{-\alpha}-\frac{(8 \pi+4 \pi \varepsilon)}{\xi \alpha} \frac{2 \eta}{\left(1+\frac{2 \alpha}{3}-\frac{\alpha^{2}}{12} \omega\right)} \\
& =C a^{-\alpha}-\Psi
\end{aligned}
$$

where

$$
C=\frac{(8 \pi+4 \pi \varepsilon)}{6 \xi} \frac{\left(1+\frac{12 \eta}{\alpha}\right)}{\left(1+\frac{2 \alpha}{3}-\frac{\alpha^{2}}{12} \omega\right)}
$$

and

$$
\Psi=\frac{(8 \pi+4 \pi \varepsilon)}{\xi \alpha} \frac{2 \eta}{\left(1+\frac{2 \alpha}{3}-\frac{\alpha^{2}}{12} \omega\right)} .
$$

Integrating equation (16) with $\alpha>0, \Psi>0$

$$
a(t)=a_{0}\left[\cos \left(\left|\frac{\alpha}{2}\right| \sqrt{\psi}\left(t-t_{0}\right)\right)+\sqrt{\frac{C}{\Psi} a_{0}^{-\alpha}-1} \cdot \sin \left(\left|\frac{\alpha}{2}\right| \sqrt{\Psi}\left(t-t_{0}\right)\right]^{\frac{2}{\alpha}}\right.
$$

and for $\alpha<0, \Psi>0$

$$
a(t)=a_{0}\left[\operatorname{Cos}\left(\left|\frac{\alpha}{2}\right| \sqrt{\Psi}\left(t-t_{0}\right)\right)-\sqrt{\frac{C}{\psi} a_{0}^{-\alpha}-1} \operatorname{Sin}\left(\left|\frac{\alpha}{2}\right| \sqrt{\Psi}\left(t-t_{0}\right)\right)\right]^{\frac{2}{|\alpha|}}
$$

The scale factor diverges continuously an infinite number of times. For each divergence we get Big Rip singularity which takes place at 


$$
T=t_{0}+\frac{2}{|\alpha| \sqrt{\Psi}} \arctan \left(\frac{C}{\Psi} a_{0}^{-\alpha}-1\right)^{\frac{-1}{2}}+\frac{2 n \pi}{|\alpha|}
$$

with life time given by

$$
T_{L}=\frac{2 \pi}{|\alpha| \sqrt{\Psi}}
$$

The scale factor can be written as

$$
a(\tau)=a_{\min }(\cos )^{\frac{-2}{|\alpha|}}
$$

with the new time $\tau$ covering the interval $\left(\frac{-\pi}{2}, \frac{\pi}{2}\right)$, where each cycling universe reaches the initial and final big rip at the extrema.

The dilation scalar field behaves accordingly like:

$$
\phi(\tau) \alpha \xi(\cos \tau)^{2}
$$

This scenario is similar to dark energy multiverse models [60] but with the major difference that in our scenario, universe is controlled by the periodic equation of state [58]

$$
\begin{aligned}
p(\tau)= & 3 \eta a^{\alpha}(\tau)-\rho(\tau) \\
& =3 \eta\left(1+\frac{4}{\alpha}\right) a_{\min }^{\alpha}(\cos \tau)^{2}-\left(1+\frac{12 \eta}{\alpha}\right)
\end{aligned}
$$

In order to satisfy restriction that Brans-Dicke parameter is time dependent, it is required to have from equation (15), the equation of state $4 p=(1+\in) \rho$. This yields $w=-3 / 2$, which is the same value obtained in conformal relativity action within the framework of Brans-Dicke cosmology, in compare to the reduced (gravity-dilaton) low-energy-effective superstring action which corresponds to a Brand-Dicke action with Brans-Dicke parameter $\omega \approx-1$. In fact, Brans-Dicke parameter $\omega \approx-3 / 2$ gives a margin between a standard scalar field and a ghost.

Comparing (21) and the constraint $4 p=(1+\varepsilon) \rho$, we obtain

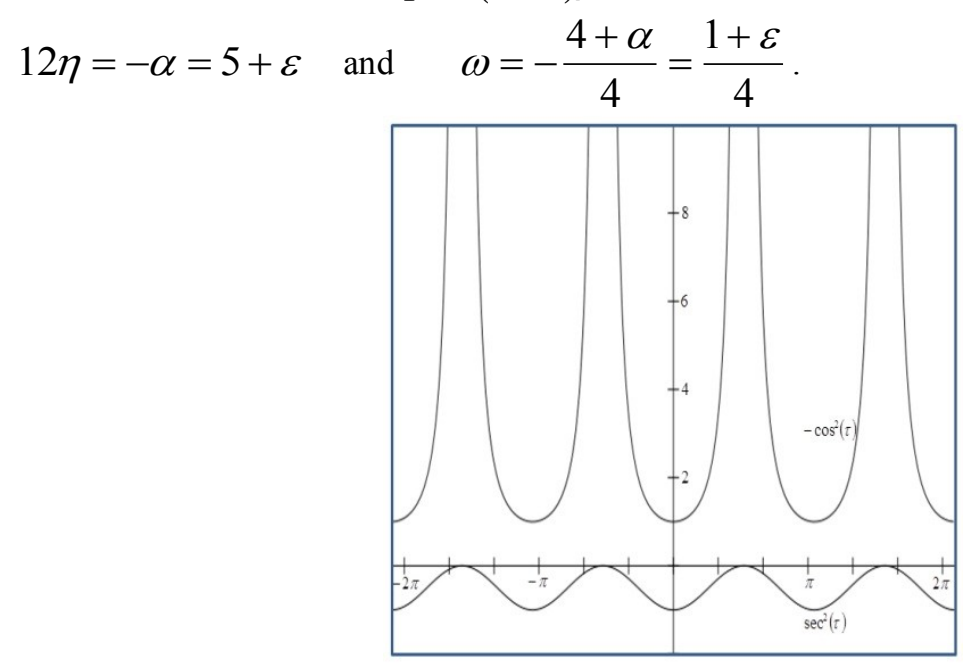

Fig. 1. Variation of the scale factor and the scalar field with $\tau$ 


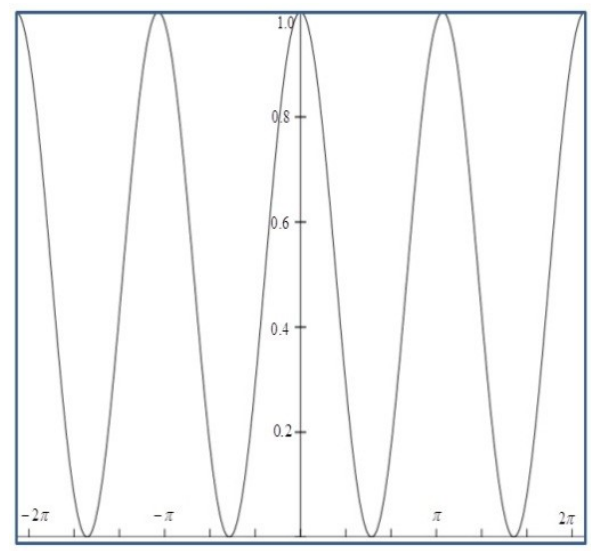

Fig. 2. Variation of the cosmological constant with $t$

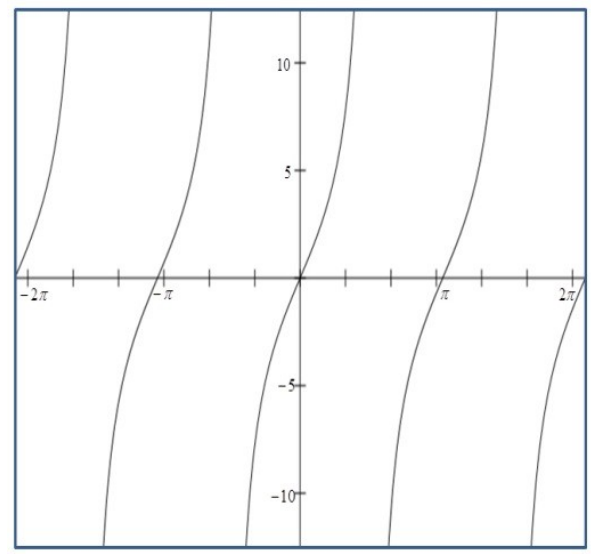

Fig. 3. Variation of the Hubble parameter $H=2 \tan \tau$ with $\tau$ for $\varepsilon=-3$

Therefor for $\varepsilon<-1$, the universe is dominated by dark energy and cosmological constant $\Lambda=\frac{4 \pi \rho \rho a^{-\alpha}}{\xi}$ is positive unless $\varepsilon<0$, i.e. negative scalar field. To illustrate, choose $\varepsilon=-3$ and $\xi=-1$ : therefore $\omega=\frac{-1}{2}$. The scenario corresponds then to a dark energy multiverse with positive cosmological constant and eternally repeating Big Rip. The scale factor and scalar field vary respectively like; $a(\tau)=a_{\text {min }}(\cos )^{-2}$ and $\phi(\tau) \alpha-(\cos \tau)^{2}$. We may plot Figures 1, 2 and 3 .

If for instance, choose $\varepsilon=-7$ and $\xi=-1$ then $\omega=-\frac{3}{2}<-1$ and multiverse is then dominated by phantom energy. For this particular choice, we find $\alpha=3$. The scenario corresponds in that case to a phantom energy multiverse with positive cosmological constant and eternally repeating Big Rip. The scale factor varies like $a(\tau)=a_{\min }(\cos )^{\frac{-2}{3}}$ whereas the scalar field behaves like $\phi(\tau) \alpha-(\cos \tau)^{2}$. The Hubble parameter is $H=2 \tan \frac{\tau}{3}$ and is as well periodic.

\section{Conclusion:}

A dark energy and phantom energy dilation Brans-Dicke multiverse with positive cosmological constant has been investigated by El Nabulsi (2010), whose work has been extended and studied in five dimensions. An attempt has been made to retain El-Nabulsi (2010) form of the various quantities. The model is controlled by generalized equation of state $\quad p=3 \eta a^{\alpha}-\rho$, a cosmological constant behaving like $\Lambda \phi=4 \pi \varepsilon \rho$ and a dilation scalar field behaving like $\phi=\xi a^{\alpha}$, where $12 \eta=-\alpha=(\varepsilon+5)$ and $\varepsilon<0$. It 
is quite interesting to obtain a higher dimensional cosmological multiverse model in which the lambda is positive and not negative, in contrast to those models that rise from string, superstring and supergravity models where the vacuum has negative energy density and hence describe by an AdS space time. As we have $|G| \alpha \phi^{-1}$ , then obviously the gravitational coupling constant is well oscillatory in our scenario. We have note that all the results of El Nabulsi (2010) can be obtained from our results by assuming appropriate values of the functions concerned.

[1] Riess, A. G. et al., (1998). Observational Evidence from Supernovae for an Accelerating Universe and a Cosmological Constant. Astron. J. 1161009.

[2] Riess, A. G. et al. (2004). Type Ia Supernova Discoveries at $\mathrm{z}>1$ from the Hubble Space Telescope: Evidence for Past Deceleration and Constraints on Dark EnergyEvolution1. Astrophys. J. 607665.

[3] Knop, R. A. et al. (2003). New Constraints on $\Omega \mathrm{M}, \Omega \Lambda$, and $\mathrm{w}$ from an Independent Set of 11 High-Redshift Supernovae Observed with the Hubble Space Telescope. Astrophysics J. 598102.

[4] Gasperini, M., . Veneziano, G. (2003). The pre-big bang scenario in string cosmology. Phys. Rep. 373 1-2.

[5] Perlmutter, S., et al.(1999). Measurements of $\Omega$ and $\Lambda$ from 42 High-Redshift Supernovae Astrophys. J. 517565.

[6] Peebles, P. J. E. and Ratra, B. (2003). The cosmological constant and dark energy. Rev. Mod. Phys. 75559.

[7] Bennett, C. L., et al. (2003). First-Year Wilkinson Microwave Anisotropy Probe (WMAP) Observations: Preliminary Maps and Basic Results. Astrophys. J., Suppl. Ser. 1481 .

[8] Spergel, D. N., et al. (2003). First-Year Wilkinson Microwave Anisotropy Probe (WMAP) Observations: Determination of Cosmological Parameters. Astrophys. J., Suppl. Ser. 148175.

[9] Allen, S. W., Schmidt, R. W., Ebeling, H. Fabian, A. C., Van Speybroeck, L. (2004). Constraints on dark energy from Chandra observations of the largestrelaxed galaxy clusters. Mon. Not R. Astron. Soc. 353457.

[10] Fabris, J. C., Concalves, S. V. B., de. Souza, P. E.(2002). Density Perturbations in a Universe Dominated by the Chaplygin Gas. Gen. Rel. Grav., 34, (1) 53 .

[11] Fabris, J. C. et al., (2007). Constraining a double component dark energy model using supernova type Ia data. Phys. Lett. A 367 423.

[12] Bento, N. C., Bertolami, O., Sen, A. A. (2002). Generalized Chaplygin gas, accelerated expansion, and dark-energy-matter unification. Phys. Rev. D 66043507.

[13] Setare, M. R. (2006 a ). Generalized second law of thermodynamics in quintom dominated universe Phys. Lett. B 641130.

[14] Setare, M. R. (2006 b). Interacting holographic dark energy model in non-flat universe Phys. Lett. B $\mathbf{6 4 2} 1$.

[15] Setare, M. R. (2007). Generalized Chaplygin gas, accelerated expansion, and dark-energy-matter unification . Phys. Lett. B. 644 (2-3) 99 .

[16] Setare, M. R. and Saridakis, M., (2008 a). The quintom model with $\mathrm{O}(\mathrm{N})$ symmetry. J. Cosmol. Astropart. Phys. 0809026.

[17] Setare, M. R. and Saridakis, M.(2008 b). Coupled oscillators as models of quintom dark energy .Phys. Lett. B. 668 (3) 177.

[18] Witten, (1981). Some properties of O(32) superstrings. Phy. Lett., B. 149 (4-5) 351.

[19] Alvarez, E., Gavela, M. B. (1983). Entropy from Extra Dimensions. Phys. Rev. Lett. 51931.

[20] Randjbar-Daemi, S. S., Strathdec, A. (1984). On Kaluza-Klein cosmology. J. Phys. Lett. B,135 (5-6) 388.

[21] Macriano, W. J. (1984). Time Variation of the Fundamental "Constants" and Kaluza-KleinTheories. Phys. Rev. Letts. 52489.

[22] Kaluza T. Sitz. Preuss. (1921). Zum Unitätsproblem in der Physik. Acad. Wiss. Phys. Mth., KI. 966.

[23] Klein O .Z. (1926). Quantentheorie und fünfdimensionale Relativitätstheorie . Phys.,37 (12), 895.

[24] Thiry Y, C. R. (1948). Acad. Sci. (Paris), 226, 216.

[25] Jordon, P. (1959). Zum gegenwärtigen Stand der Diracschen kosmologischen Hypothesen. Z. Phys. 157112.

[26] Chatterjee, S., Bhui, B. (1990). Mon. Not. R. Astr. Soc., 247, 57.

[27] Banerjee, A., Bhui, B. K. (1990). Astron. Astrophys., 232, 305.

[28] Singh, G. P., Kotambkar, S.(2001). Higher Dimensional Cosmological Model With Gravitational and Cosmological "Constants. Gen. Rel. Grav , 33, 621 .; Gravit. \& Cosm., 9, 206 (2003) ; Pramana, 35, (2005).

[29] Reddy D. R. K., Venkateswara Rao, N. (2001). Some Cosmological Models in Scalar-Tensor Theory of Gravitation. Astro. Phys. Space Sci. 277461.

[30] Chakravarty N., Charkarborty S., Santra B. C.(2003). Higher Dimensional space-time model for a dust filled universe with a cosmological constant. Int. J. Mod. Phys. D 12 (5) 905.

[31] Singh G. P.,. Kotambkar S., Pradhan, A. (2003). Higher Dimensional Cosmological Model in Lyra Geometry. Int. J. Mod. Phys. D 12853.

[32] Paul S. Wesson (2004). Space-Time Uncertainty from Higher-Dimensional Determinism. Gen. Rel . Grav. 36451.

[33] Adhav, K. S., Nimkar, A. S., Dawande, M . V.(2007). N-dimensional string cosmological model in Brans-Dicke theory of gravitation. Astrophys. Spa. Sci. 310 231-235.

[34] Katore ,S. D., Shaikh A. Y., Kapase D. K., Bhaskar S. A. (2001). FRW Bulk Viscous Cosmology in Multi-Dimensional SpaceTime. Int. J. Theo. Phys., 50 2644-2654.

[35] Brans, C., Dicke, R. H. (1961). Mach's Principle and a Relativistic Theory of Gravitation Phys. Rev. 124925.

[36] Weinberg, S.(wiley,1972). Principles and applications of the general theory of relativity. Grav. and Cosm.

[37] Nariai, H. (1972). Hamiltonian Approach to the Dynamics of Expanding Homogeneous Universes in the Brans-Dicke Cosmology. Progress of Theoretical Physics. 47 (6) 1824-1843.

[38] Belinskii, V. A. and Khalatnikov, I. M. (1973). Effect of Scalar and Vector Fields on the Nature of the Cosmological Singularity . Soviet Physics JETP, 36 (4) 591-597.

[39] Reddy, D. R. K. and Rao, V. U. M. (1981). Field of a charged particle in Brans-Dicke theory of gravitation . Mathematical and General. 148 1973-1976.

[40] Banerjee, A. and Santos, N. O. (1982). Bianchi type-II cosmological models in Brans-Dicke theory. Nuovo Cimento B 67 (1) $31-40$.

[41] Singh, T., Rai. L. N. and Singh T.(1983). An anisotropic cosmological model in Brans-Dicke theory. Astrophysics and Space Science, 96 (1) 95-105. 
[42] Ram, S. (1983). Spatially homogeneous and anisotropic cosmological solution in Brans-Dicke theory Gravitation 15 (7) 635-640.

[43] Ram, S., Singh, D. K. (1984). LRS Bianchi type V vacuum cosmological solution in Brans-Dicke theory. Astro-physics and Space Science, 98 (1) 193- 196.

[44] Berman, M. S., Som M. M. and Gomide F. M.(1989). Brans-Dicke static universes. General Relativity and Gravitation. 21 (3) 287-292.

[45] Reddy, D. R. K., (2003). A string cosmological model in a scalar - Tensor theory of gravitation. Astrophysics and Space Science . 286 (3-4) 359-363.

[ 46] Reddy, D. R. K. and. Naidu R. L.(2007). Five dimensional string cosmological models in a scalar-tensor theory of gravitation Astrophysics and Space Science $\mathbf{3 0 7}$ (4) 395-398.

[47] Adhav, K. S., Nimkar A. S. and Dawande M. V. (2007). N-dimensional string cosmological model in Brans-Dicke theory of gravitation. Astrophysics and Space Science $\mathbf{} 310$ (3-4) 231-235.

[48] Rao, V. U. M., Vinutha T., Shanthi M. V. and Sireesha K. V. S. (2008). Exact Bianchi type-V perfect fluid cosmological models in Brans-Dicke theory of gravitation. Astrophys. and Spa. Sci. 315 (1-4) 211-214.

[49] El-Nabulsi , A.R. (2008 a). Effective 3-Brane Brans-Dicke Cosmology. Mod. Phys. Lett. A .23 (6) 401.

[50] El- Nabulsi.A.R. (2008 b). Accelerated D-Dimensional Compactified Universe in Gauss--Bonnet--Dilatonic Scalar Gravity from D-Brane M-Theory. Chin.Phys.Lett. 25 (8) 2785.

[51] El- Nabulsi , A.R .2009 a). Maxwell Brane Cosmology with Higher-Order String Curvature Corrections, a Non-Minimally Coupled Scalar Field Dark Matter-Dark Energy Interaction and a Varying Speed of Light. Int. J. Mod. Phys. D 18 (2) 289.

[52] El- Nabulsi, A. R. (2009 b). Modified Brane World Cosmoilogies in Presence of Stringy Corrections Cpoupled to a Canonical Scalar Field. Int. J. Mod. Phys. D 18 (15) 691.

[53] El- Nabulsi ,A.R.(2009 c). Accelerated cosmic expansion from Gauss-Bonnet curvature corrections, nonminimal coupling, and infrared second-order gauge gravity. Astrophy. Spa. Sci. 325 (2) 149.

[54] El- Nabulsi ,A.R.(2009 d). Quintessence cosmology with a traversable wormhole, massive decaying gravitons and with varying G and $\Lambda$. Astrophys. Spa. Sce. $325(2), 277$.

[55] El- Nabulsi ,A. R. (2009 e). Accelerated expansion of the universe in a non-trivial extra-dimensional topology. Astrophys. Spa. Sci. 324 (1) 71

[56] El- Nabulsi, A. R. (2009 f). Astrophys. Spa. Sci. doi.10.1007/s10509

[57] El- Nabulsi, A. R. (2009 g). Acclerated Magnetic Gauss-Bonnet Cosmology. Fizika B. 17 (4) 455.

[58] El- Nabulsi , A. R. (2010). A dark energy and phantom energy dilaton-Brans-Dicke multiverse with positive cosmological constant. Astro. Phys. Spa. Sci, 327 (2) 155-159.

[59] Mukhopadhay, U., Ghosh, P. P., Ray, S. (2010). Higher Dimensional Dark Energy Investigation with Variable \$ $\$$ and $\$$ G\$. arXiv:1001.0475.

[60] Robles-Perez .S, Martin-Moruno.P., Rozas-Fernendez. A. GonzalezDiaz. P.( 2007). A Dark Energy Multiverse. Class. Quamtum Grav. 24 F41. 